\title{
Constrains of Charge-to-Mass Ratios on Noncommutative Phase Space
}

\author{
Kai Ma \\ School of Physics Science, Shaanxi University of Technology, Hanzhong, Shaanxi 723000, China \\ Correspondence should be addressed to Kai Ma; makainca@yeah.net
}

Received 26 June 2017; Revised 9 October 2017; Accepted 25 October 2017; Published 16 November 2017

Academic Editor: Yandong Liu

Copyright (c) 2017 Kai Ma. This is an open access article distributed under the Creative Commons Attribution License, which permits unrestricted use, distribution, and reproduction in any medium, provided the original work is properly cited. The publication of this article was funded by SCOAP ${ }^{3}$.

\begin{abstract}
Based on recent measurements on the charge-to-mass ratios of proton and antiproton, we study constraints on the parameters of noncommutative phase space. We find that while the limit on the parameter of coordinate noncommutativity is weak, it is very strong on the parameter of momentum noncommutativity, $\sqrt{\xi} \lesssim 1 \mu \mathrm{eV}$. Therefore, the charge-to-mass ratio experiment has a strong sensitivity on the momentum noncommutativity, and enhancement of future experimental achievement can further pin down the momentum noncommutativity.
\end{abstract}

\section{Introduction}

The noncommutative filed theories are established on a noncommutative space which is characterized by a deformed algebra between coordinate operators,

$$
\left[x_{\mu}, x_{\nu}\right]=i \theta_{\mu \nu}
$$

and parameterized by the totally antisymmetric constant ten$\operatorname{sor} \theta_{\mu \nu}$ which has dimension of length-squared. Such a model was originally proposed to address the infinity problem in quantum field theory $[1,2]$ and was shown later that similar property can also appear in both string theory embedded in a background magnetic field [3] and quantum gravity [4]. It has been shown that the rotational symmetry can be broken $[5,6]$, and consequently the energy levels of hydrogen atom [7] and Rydberg atoms [8] and topological phase effects [9-11] as well as the quantum speed of relativistic charged particles [12-15] and fluid [16] can receive interesting corrections. Algebra (1) can be accomplished by a replacement $x_{\mu} \rightarrow$ $x_{\mu}+p_{\mu} /(2 \hbar)$. However, it has been pointed out that this simple shift method can not lead to gauge invariant results $[17,18]$, and the nontrivial gauge invariant physical effects exist only for the noncommutative algebra in the momentum space described as follows [19-26]:

$$
\left[p_{\mu}, p_{\nu}\right]=i \xi_{\mu \nu}
$$

where $\xi_{\mu \nu}$ is also a totally antisymmetric constant tensor and parameterizing the momentum noncommutativity. In consideration of that the momentum operators are defined as the derivatives of the action with respect to the noncommutative coordinates, algebra (2) can appear naturally as a result of algebra (1). A more general investigation on the whole Poincare group was conducted recently in [27] and relativistic corrections to the algebra of position variables and spinorbital interaction were studied in [28]. Therefore, in case that the gauge problem can be cured by using the SeibergWitten (SW) map $[3,9,10]$, it is interesting to study the physical effects when both the nontrivial algebras (1) and (2) exist.

On the other hand, noncommutative field theories can invalidate the CPT theorem [29-32], which is one of the most profound symmetry implied in any local and Lorentz invariant field theory [33]. Generally, the CPT invariance can be broken in extended field theories with either broken [34-38] or conserved Lorentz symmetry [29, 30, 39]. The noncommutative extensions are of particular interesting 
since the CPT symmetry can be broken in both ways [29-31] and has been extensively studied [29-32].

In this paper, we study the constraint on the noncommutative parameters $\theta$ and $\xi$ by using recent experimental results on the charge-to-mass ratio of proton and antiproton [40]. The CPT invariance implies proton and antiproton have completely the same charge-to-mass ratios, apart from a sign. The measurement in [40] gives a strong limit on possible derivation

$$
\frac{(Q / m)_{\bar{p}}}{(Q / m)_{p}}-1=1(69) \times 10^{-12} .
$$

Due to the fact that the noncommutativities of phase space are purely geometrical properties, its physical effects are independent of composition of the particle. The measurements on proton and antiproton in [40] are expected to give strong constraints on the noncommutative parameters.

The contents of this paper are organized as follows: in Section 2 we study the noncommutative corrections on cyclotron frequency of a charged particle in an external magnetic field; in Section 3 we study the constraints of the results in [40] on noncommutative parameters; in Section 4 we study the constraints of the results in [40] on a related model with Lorentz violation; summary is given in Section 5 .

\section{Noncommutative Corrections on Cyclotron Frequency}

In general, there are two distinct proper "fundamental" representations for matter fields under the noncommutative $U(1)$ group [31]. The first one which is called the positive representation has a gauge transformation $\psi_{+}^{\prime}=U(x) \star \psi_{+}$, while the second one which is called the negative representation is $\psi_{-}^{\prime}=\psi_{-} \star U^{-1}(x)$, where the $\star$-product is a realization of algebra (1). And for every one, there is a corresponding "covariant" derivative

$$
\begin{aligned}
& D_{\mu}^{+} \psi=\partial_{\mu} \psi+i e \psi \star A_{\mu}, \\
& D_{\mu}^{-} \psi=\partial_{\mu} \psi-i e A_{\mu} \star \psi .
\end{aligned}
$$

With each of the covariant derivatives defined above, the Lagrangian,

$$
\mathscr{L}^{ \pm}=\overline{\psi_{ \pm}} \star\left(i \gamma^{\mu} D_{\mu}^{ \pm}-m\right) \star \psi_{ \pm},
$$

is invariant under the noncommutative $U(1)$ transformations. These two types of fermions are related by a charge conjugation transformation [31]. With the assumption that the noncommutative parameter reverses its sign, that is $\theta \rightarrow$ $-\theta$, under $C$ transformation, then the noncommutative quantum electrodynamics (NCQED) preserves $C$ symmetry. Even through the above $\theta$ transformation property has an intuitive explanation [31, 41,42], it is more interesting to investigate the phenomenology of the $C$ violating NCQED in consideration of the fact that in this case even neutral particles can couple to photons $[43,44]$.

No matter which representation is chosen, the SW map [3] can be employed to keep the original gauge symmetry $[43,44]$. It has also been shown that the coordinate and momentum noncommutatives can appear simultaneously in a consistent way in which the ordinary electromagnetic gauge symmetry can be preserved $[25,26]$, and the Lagrangian density for charged particle interacting with external electromagnetic fields has been obtained as follows:

$$
\mathscr{L}=\bar{\psi}(x)\left(\not p-Q A_{\mathrm{NC}}-m\right) \psi(x),
$$

where $Q$ is the charge of matter particle in unit of $|e|$ and the effective potential $A_{\mathrm{NC} ; \mu}=A_{\mu}+A_{\xi ; \mu}$, that is, a sum of the original one $A_{\mu}$ and an effective term $A_{\xi ; \mu}$ emerging due to the noncommutativity of momenta operators and having the following expression:

$$
A_{\xi ; \mu}=\frac{\xi}{2 \hbar Q}(0, y,-x, 0)
$$

It should be stressed that the above expression of $A_{\xi ; \mu}$ is obtained by defining the $\widehat{z}$-axis as the direction of the vector $\vec{\xi}$ whose components are required to relate to the noncommutative parameter $\theta_{i j}$ by the relation $\xi_{i}=\epsilon_{i j k} \xi^{i j} / 2$ such that nonzero components are only $\xi^{12}=-\xi^{21}=\xi$. In this configuration, the effect of momentum-momentum noncommutativity is an addition of a constant magnetic background field $\vec{B}_{\xi}=\vec{\nabla} \times \vec{A}_{\xi}=\left(0,0, B_{\xi}\right)$, with $B_{\xi}=\xi /(\hbar Q)$ over the whole space. The nonrelativistic approximation can be obtained by using the well-known Foldy-Wouthuysen unitary transformation (FWUF) [45] and by neglecting the spin degree of freedom the nonrelativistic Hamiltonian is given as $[25,26]$

$$
H_{\mathrm{NC}}=\frac{1}{2 m_{\theta}}\left(\vec{p}-\overrightarrow{\mathrm{A}}_{\mathrm{NC}}\right)^{2}
$$

where the noncommutative effective mass

$$
m_{\theta}=m \alpha_{\theta}^{-1}, \quad \alpha_{\theta}=1-Q \frac{\vec{\theta} \cdot \vec{B}_{\mathrm{NC}}}{2 \phi_{0}},
$$

and $\phi_{0}=h / e$ is the fundamental magnetic flux. Because of that $|\theta||\xi| \ll \hbar^{2}$, and furthermore usually the external magnetic field $|\vec{B}|$ is much stronger than the noncommutative background $\left|\vec{B}_{\xi}\right|$; the scale factor $\alpha_{\theta}$ in (20) can be approximated as

$$
\alpha_{\theta} \approx 1-Q \frac{\vec{\theta} \cdot \vec{B}}{2 \phi_{0}}
$$

We will use this approximation in the rest of this paper to estimate the constraint on noncommutative parameters.

The charge-to-mass ratios reported in [40] were obtained by measuring the cyclotron frequency of charged particles in a constant external magnetic field $B_{0}=1.946 \mathrm{~T}$. Therefore, we need to know the dynamical properties of charged particle in a constant external magnetic field on noncommutative phase space. We require that the external magnetic field is also along the $z$ direction; that is, $\vec{B}=B \vec{e}_{z}$. This is not true in general but is a good approximation since the noncommutative 
correction is maximum in this case. On the other hand the measurement on the sidereal variations in [40], which gives an upper bound of 720 parts per trillion that is a little weaker than (3), also justifies our approximation. Furthermore, we chose the symmetric gauge to solve the static Schrödinger equation, and the ordinary gauge potential can be expressed in this gauge as $\vec{A}=(1 / 2) B(-y, x, 0)$. In consideration that the $z$ component can be factorized completely and plane wave solutions are sought, we will neglect it in the rest of this paper, and explicit expression of the transverse part of the Hamiltonian (19) can be obtained from upon expansion

$$
H_{\|}=\frac{p_{x}^{2}}{2 m_{\theta}}+\frac{1}{2} m_{\theta} \omega^{2} x^{2}+\frac{p_{y}^{2}}{2 m_{\theta}}+\frac{1}{2} m_{\theta} \omega^{2} y^{2},
$$

where the Larmor frequency $\omega=\mathrm{Q} B_{\mathrm{NC}} /\left(2 m_{\theta}\right)$. One can see that this Hamiltonian mimics the ordinary Landau problem, apart from a correction on the Larmor frequency. The noncommutative extension of the Landau system has been studied extensively [46-54]. However, so far the charge-tomass ratio related physics have not been studied. It is wellknown that the corresponding eigenvalue problem can be solved exactly in terms of polar coordinates, and the energy eigenvalues are given as

$$
E=\left(n+\frac{1}{2}\right) \hbar \omega_{C}, \quad \omega_{C}=2 \omega
$$

where $\omega_{C}$ is the cyclotron frequency.

\section{Constraints of Charge-to-Mass Ration}

The charge-to-mass ratio can be extracted from the measured cyclotron frequency and external magnetic field as follows:

$$
\left[\frac{\mathrm{Q}}{m}\right]_{\exp }=\frac{\omega_{\mathrm{C}}}{B}=\frac{\mathrm{Q}}{m} \cdot \alpha_{\theta} \cdot \frac{B_{\mathrm{NC}}}{B} .
$$

In case that noncommutative parameters are small, the noncommutative corrections can be approximated as

$$
\left[\frac{Q}{m}\right]_{\exp } \approx \frac{Q}{m}\left(1-\frac{Q \theta B}{2 \phi_{0}}+\frac{\xi}{\hbar Q B}\right) .
$$

Under this approximation, the antiproton-to-proton mass ratio is given as

$$
\chi_{\mathrm{NC}}=\frac{\left(Q / m_{\theta}\right)_{\bar{p}}}{\left(Q / m_{\theta}\right)_{p}} \approx \chi+\frac{\theta B}{\phi_{0}}-\frac{2 \xi}{e \hbar B}
$$

where $\chi=1$ due to the CPT conservation of the ordinary field theory. Therefore, by requiring that the noncommutative corrections lie in the $1 \sigma$ region of the experimental error, the result (3) given in [40] puts a constraint,

$$
\left|\frac{\theta B_{0}}{\phi_{0}}-\frac{2 \xi}{e \hbar B_{0}}\right| \leq 0.69 \times 10^{-12},
$$

on the noncommutative parameters $\theta$ and $\xi$. In case that either $\theta$ or $\xi$ vanishes, one has the following upper limits:

$$
\begin{aligned}
& \theta \leq 1.46 \times 10^{-27} \mathrm{~m}^{2}, \\
& \xi \leq 1.14 \times 10^{-65} \mathrm{~kg}^{2} \cdot \mathrm{m}^{2} \cdot \mathrm{s}^{-2} .
\end{aligned}
$$

While the limit on the noncommutative parameter $\theta$ is not strong, $1 / \sqrt{\theta} \gtrsim 0.5 \mathrm{MeV}$ in unit of energy and the constraint on the noncommutative parameter $\xi$ is very strong, $\sqrt{\xi} \lesssim 1 \mu \mathrm{eV}$. Therefore, the charge-to-mass ratio experiment performed in [40] has a strong sensitivity on the momentum noncommutativity.

\section{Constraint on Lorentz Violation Parameter}

It has been pointed out that the noncommutative extension of quantum field theory can be effectively described by a quantum field theory with Lorentz violation $[55,56]$. Therefore, it is expected to give strong constraints on the Lorentz violation parameters. In this section, we study constraint of charge-tomass ratio on the Lorentz violation.

In general there can be a lot of parameters in a quantum field theory with Lorentz violation $[57,58]$. Here we consider only the following Lagrangian:

$$
\mathscr{L}=\bar{\psi}\left(i \gamma^{\mu} D_{\mu}+i Q c^{\mu \nu} \gamma_{\mu} D_{\nu}-m\right) \psi,
$$

where $c^{\mu \nu}$ is a constant tensor, parameterizing the strength of Lorentz violation. The CPT invariance is explicitly violated by the charge dependence of the anomalous interaction term. The measurement in [40] is expected to give a strong limit on the parameter $c^{\mu \nu}$. The nonrelativistic approximation can be obtained by using the well-known Foldy-Wouthuysen unitary transformation (FWUF) [45], and neglecting the spin degree of freedom the nonrelativistic Hamiltonian is given as [59]

$$
H=\frac{1}{2 \widetilde{m}}(\vec{p}-Q \vec{A})^{2}
$$

where the effective mass

$$
\widetilde{m}=m\left(1+Q c_{00}\right) .
$$

By neglecting the trivial dynamics along $z$ direction, the explicate expression of the transverse part of the Hamiltonian (19) is

$$
H_{\|}=\frac{p_{x}^{2}}{2 \widetilde{m}}+\frac{1}{2} \widetilde{m} \omega^{2} x^{2}+\frac{p_{y}^{2}}{2 \widetilde{m}}+\frac{1}{2} \widetilde{m} \omega^{2} y^{2},
$$

where the Larmor frequency $\omega=Q B /(2 \widetilde{m})$. One can see that this Hamiltonian mimics the ordinary Landau problem. The eigenvalue problem can then be solved exactly in terms of polar coordinates, and the energy eigenvalue is given as follows:

$$
E=\left(n+\frac{1}{2}\right) \hbar \omega_{C}, \quad \omega_{C}=2 \omega,
$$

where $\omega_{C}$ is the cyclotron frequency. 
The charge-to-mass ratio can be extracted from the measured cyclotron frequency and external magnetic field as follows:

$$
\left[\frac{Q}{m}\right]_{\exp }=\frac{\omega_{C}}{B}=\frac{Q}{m\left(1+Q c_{00}\right)} .
$$

In case that parameters $c_{00}$ are small, the corrections can be approximated as

$$
\left[\frac{Q}{m}\right]_{\exp } \approx \frac{Q}{m}\left(1-Q c_{00}\right) .
$$

In this approximation, the antiproton-to-proton mass ratio is given as

$$
\tilde{\chi}=\frac{\left(Q / m_{\theta}\right)_{\bar{p}}}{\left(Q / m_{\theta}\right)_{p}} \approx \chi+2 c_{00}
$$

where $\chi=1$ due to the CPT conservation of the ordinary field theory. Therefore, by requiring that the corrections lie in the $1 \sigma$ region of the experimental error, result (3) given in [40] gives the following constraint:

$$
\left|c_{00}\right| \leq 0.345 \times 10^{-12} .
$$

\section{Summary}

In summary, we study the quantum properties of a charged particle in a constant external magnetic field, and by using the recent measurement on the charge-to-mass ratios of proton and antiproton [40], we have shown that while the chargeto-mass ratio experiment is not sensitive to the parameter of coordinate noncommutativity, it can give strong constraint on the parameter of momentum noncommutativity. The current bound is $\sqrt{\xi} \leqslant 1 \mu \mathrm{eV}$ (in unite of energy). It is expected that future enhancement of experimental precision can further pin down the momentum noncommutativity. We also studied related model with Lorentz violation.

\section{Conflicts of Interest}

The author has no conflicts of interest.

\section{Acknowledgments}

Kai Ma is supported by the China Scholarship Council and the National Natural Science Foundation of China under Grants nos. 11647018 and 11705113.

\section{References}

[1] H. S. Snyder, "Quantized space-time," Physical Review A: Atomic, Molecular and Optical Physics, vol. 71, pp. 38-41, 1947.

[2] C. N. Yang, "On quantized space-time," Physical Review, vol. 72, article 874, 1947.

[3] N. Seiberg and E. Witten, "String theory and noncommutative geometry," Journal of High Energy Physics, vol. 1999, no. 09, article 032, 1999.
[4] L. Freidel and E. L. Livine, "3D quantum gravity and effective noncommutative quantum field theory," Physical Review Letters, vol. 96, no. 22, Article ID 221301, 4 pages, 2006.

[5] M. R. Douglas and N. A. Nekrasov, "Noncommutative field theory," Reviews of Modern Physics, vol. 73, no. 4, pp. 977-1029, 2001.

[6] R. J. Szabo, "Quantum field theory on noncommutative spaces," Physics Reports, vol. 378, no. 4, pp. 207-299, 2003.

[7] M. Chaichian, M. M. Sheikh-Jabbari, and A. Tureanu, "Hydrogen atom spectrum and the lamb shift in noncommutative QED," Physical Review Letters, vol. 86, no. 13, pp. 2716-2719, 2001.

[8] J. Zhang, "Testing Spatial Noncommutativity via Rydberg Atoms," Physical Review Letters, vol. 93, no. 4, article 043002, 2004.

[9] K. Ma, J. Wang, and H. Yang, "Seiberg-Witten map and quantum phase effects for neutral Dirac particle on noncommutative plane," Physics Letters B, vol. 756, pp. 221-227, 2016.

[10] K. Ma, J.-H. Wang, and H.-X. Yang, "Time-dependent Aharonov-Bohm effect on the noncommutative space," Physics Letters B, vol. 759, pp. 306-312, 2016.

[11] M. A. Anacleto, F. A. Brito, A. Mohammadi, and E. Passos, "Aharonov-Bohm effect for a fermion field in a planar black hole "spacetime"," The European Physical Journal C, vol. 77, no. 4, article 239, 2017.

[12] K. Wang, Y.-F. Zhang, Q. Wang, Z.-W. Long, and J. Jing, "Quantum speed limit for a relativistic electron in the noncommutative phase space," International Journal of Modern Physics A, vol. 32, no. 23-24, Article ID 1750143, 2017.

[13] K. Wang, Y. Zhang, Q. Wang, Z. Long, and J. Jing, "Quantum Speed Limit for Relativistic Spin-0 and Spin-1 Bosons on Commutative and Noncommutative Planes," Advances in High Energy Physics, vol. 2017, Article ID 4739596, 9 pages, 2017.

[14] A. A. Deriglazov and W. G. Ramírez, "Mathisson-PapapetrouTulczyjew-Dixon equations in ultra-relativistic regime and gravimagnetic moment," International Journal of Modern Physics D, vol. 26, no. 6, Article ID 1750047, 2017.

[15] A. A. Deriglazov and W. G. Ramírez, "Ultrarelativistic spinning particle and a rotating body in external fields," Advances in High Energy Physics, vol. 2016, Article ID 1376016, 27 pages, 2016.

[16] P. Das and S. Ghosh, "Noncommutative geometry and fluid dynamics," The European Physical Journal C, vol. 11, article 627, (11) (2016), [Erratum: The European Physical Journal C, vol. 77, no. 2 , article 64,2017$]$.

[17] M. Chaichian, M. Långvik, S. Sasaki, and A. Tureanu, "Gauge covariance of the Aharonov-Bohm phase in noncommutative quantum mechanics," Physics Letters B, vol. 666, no. 2, pp. 199204, 2008.

[18] O. Bertolami and P. Leal, "Aspects of phase-space noncommutative quantum mechanics," Physics Letters B, vol. 750, pp. 6-11, 2015.

[19] E. Langmann and R. J. Szabo, "Duality in scalar field theory on noncommutative phase spaces," Physics Letters B, vol. 533, no. 1-2, pp. 168-177, 2002.

[20] E. Langmann, R. Szabo, and K. Zarembo, "Exact solution of quantum field theory on noncommutative phase spaces," Journal of High Energy Physics, vol. 2004, no. 1, article 017, 2004.

[21] K. Li and N. Chamoun, "Hydrogen atom spectrum in noncommutative phase space," Chinese Physics Letters, vol. 23, pp. 1122$1123,2006$. 
[22] K. Li and J. Wang, "The Topological AC effect on noncommutative phase space," The European Physical Journal, vol. 50, pp. 1007-1011, 2007.

[23] B. Muthukumar, "Remarks on the formulation of quantum mechanics on noncommutative phase spaces," Journal of High Energy Physics, vol. 2007, no. 1, article 073, 2007.

[24] S. Liang, H. Li, and G. Huang, "Detecting noncommutative phase space by the Aharonov-Bohm effect," Physical Review A: Atomic, Molecular and Optical Physics, vol. 90, no. 1, Article ID 010102, 2014.

[25] K. Ma, Y.-J. Ren, Y.-H. Wang, and K.-Q. Liu, "Probing noncommutativities of phase space by using persistent charged current and its asymmetry," https://arxiv.org/abs/1703.10923.

[26] K. Ma, J. H. Wang, and H. X. Yang, "Probing the noncommutative effects of phase space in the time-dependent AharonovBohm effect," Annals of Physics, vol. 383, pp. 120-129, 2016.

[27] S. Meljanac, D. Meljanac, F. Mercati, and D. Pikutić, "Noncommutative spaces and Poincaré symmetries," Physics Letters B, vol. 766, pp. 181-185, 2017.

[28] A. A. Deriglazov and A. M. Pupasov-Maksimov, "Relativistic corrections to the algebra of position variables and spin-orbital interaction," Physics Letters B, vol. 761, pp. 207-212, 2016.

[29] M. Chaichian, A. D. Dolgov, V. A. Novikov, and A. Tureanu, "CPT violation does not lead to violation of Lorentz invariance and vice versa," Physics Letters B, vol. 699, no. 3, pp. 177-180, 2011.

[30] M. Chaichian, K. Fujikawa, and A. Tureanu, "Lorentz-invariant CPT violation," The European Physical Journal C, vol. 73, no. 3, article 2349, 2013.

[31] M. M. Sheikh-Jabbari, "C, P, and T invariance of noncommutative gauge theories," Physical Review Letters, vol. 84, no. 23, pp. 5265-5268, 2000.

[32] M. Chaichian, K. Nishijima, and A. Tureanu, "Spin-statistics and CPT theorems in noncommutative field theory," Physics Letters B, vol. 568, no. 1-2, pp. 146-152, 2003.

[33] G. Luders, "Proof of the TCP theorem," Annals of Physics, vol. 2, pp. 1-15, 1957.

[34] J. Ellis, N. Mavromatos, and D. Nanopoulos, "CPT violation in string modified quantum mechanics and the neutral kaon system," International Journal of Modern Physics A, vol. 11, no. 08, pp. 1489-1508, 1996.

[35] J. Ellis, J. L. Lopez, N. E. Mavromatos, and D. V. Nanopoulos, "Precision tests of CPT symmetry and quantum mechanics in the neutral kaon system," Physical Review D: Particles, Fields, Gravitation and Cosmology, vol. 53, no. 7, pp. 3846-3870, 1996.

[36] V. A. Kostelecký, "Perspectives on lorentz and CPT violation," in Proceedings of the 4th Meeting, pp. 50-56, Bloomington, Ind, USA.

[37] O. W. Greenberg, "CPT violation implies violation of Lorentz invariance," Physical Review Letters, vol. 89, no. 23, Article ID 231602, 4 pages, 2002.

[38] O. M. Del Cima, D. H. T. Franco, A. H. Gomes, J. M. Fonseca, and O. Piguet, "All orders renormalizability of a Lorentz and CPT violating quantum electrodynamics," Physical Review D, vol. 85, Article ID 065023, 2012.

[39] M. Dütsch and J. M. Gracia-Bondía, "On the assertion that PCT violation implies Lorentz non-invariance," Physics Letters B, vol. 711, no. 5, pp. 428-433, 2012.

[40] S. Ulmer, C. Smorra, A. Mooser et al., "High-precision comparison of the antiproton-to-proton charge-to-mass ratio," Nature, vol. 524, no. 7564, pp. 196-199, 2015.
[41] M. Sheikh-Jabbari, "Renormalizability of the supersymmetric Yang-Mills theories on the noncommutative torus," Journal of High Energy Physics, vol. 1999, no. 6, article 015, 1999.

[42] M. Sheikh-Jabbari, "Open strings in a B-field background as electric dipoles," Physics Letters B, vol. 455, no. 1-4, pp. 129-134, 1999.

[43] C.-b. Luo, F.-y. Hou, Z.-f. Cui, X.-j. Liu, and H.-s. Zong, "Noncommutative field with constant background fields and neutral fermions," Physical Review D: Particles, Fields, Gravitation and Cosmology, vol. 91, no. 3, Article ID 036009, 8 pages, 2015.

[44] W. Wang, Z. Yan, R. Guan, and X. Wei, "Electric dipole moment induced by $\mathrm{CP}$-violating deformations in the noncommutative Standard Model," Modern Physics Letters A, vol. 32, no. 07, Article ID 1750044, 2017.

[45] L. L. Foldy and S. A. Wouthuysen, "On the dirac theory of spin 1/2 particles and its non-relativistic limit," Physical Review A: Atomic, Molecular and Optical Physics, vol. 78, no. 1, pp. 29-36, 1950.

[46] P. Horváthy, “The Non-commutative Landau Problem," Annals of Physics, vol. 299, no. 1, pp. 128-140, 2002.

[47] S. Dulat and K. Li, "Landau problem in noncommutative quantum mechanics," Chinese Physics C, vol. 32, no. 2, pp. 9295, 2008.

[48] P. R. Giri and P. Roy, "The non-commutative oscillator, symmetry and the Landau problem," The European Physical Journal C, vol. 57, no. 4, pp. 835-839, 2008.

[49] P. D. Alvarez, J. L. Cortés, P. A. Horváthy, and M. S. Plyushchay, "Super-extended noncommutative Landau problem and conformal symmetry," Journal of High Energy Physics. A SISSA Journal, vol. 3, article 034, 2009.

[50] M. Maceda and A. Macías, "Noncommutative Landau problem in Podolsky's generalized electrodynamics," Physical Review D: Particles, Fields, Gravitation and Cosmology, vol. 79, no. 8, Article ID 087703, 2009.

[51] G. Fiore and L. Gouba, "Class of invariants for the twodimensional time-dependent Landau problem and harmonic oscillator in a magnetic field," Journal of Mathematical Physics, vol. 52, no. 10, Article ID 103509, 2011.

[52] X. Luo, Y.-S. Wu, and Y. Yu, "Noncommutative Chern-Simons theory and exotic geometry emerging from the lowest Landau level," Physical Review D: Particles, Fields, Gravitation and Cosmology, vol. 93, no. 12, Article ID 125005, 12 pages, 2016.

[53] S. Gangopadhyay, A. Saha, and A. Halder, "On the Landau system in noncommutative phase-space," Physics Letters A, vol. 379, pp. 2956-2961, 2015.

[54] X. Diao, C. Long, B. Kong, and Z. Long, "The Noncommutative Landau Problem in Podolsky's Generalized Electrodynamics," Chinese Physics Letters, vol. 32, no. 4, Article ID 040301, 2015.

[55] M. Chaichian, P. Kulish, K. Nishijima, and A. Tureanu, "On a lorentz-invariant interpretation of noncommutative space-time and its implications on noncommutative QFT," Physics Letters B, vol. 604, no. 1-2, pp. 98-102, 2004.

[56] S. M. Carroll, J. A. Harvey, V. A. Kostelecký, C. D. Lane, and T. Okamoto, "Noncommutative field theory and Lorentz violation," Physical Review Letters, vol. 87, no. 14, Article ID 141601, 2001.

[57] V. Husain and J. Louko, "Low energy Lorentz Violation from modified dispersion at high energies," Physical Review Letters, vol. 116, no. 6, Article ID 061301, 2016.

[58] A. P. Balachandran, S. Kürkçüoglu, A. R. de Queiroz, and S. Vaidya, "Spontaneous Lorentz violation: the case of infrared 
QED," The European Physical Journal C, vol. 75, no. 2, article 89, 2015.

[59] B. Gonçalves, M. M. Dias Júnior, and B. J. Ribeiro, "Exact FoldyWouthuysen transformation for a Dirac theory with the complete set of," Physical Review D: Particles, Fields, Gravitation and Cosmology, vol. 90, no. 8, Article ID 085026, 2014. 

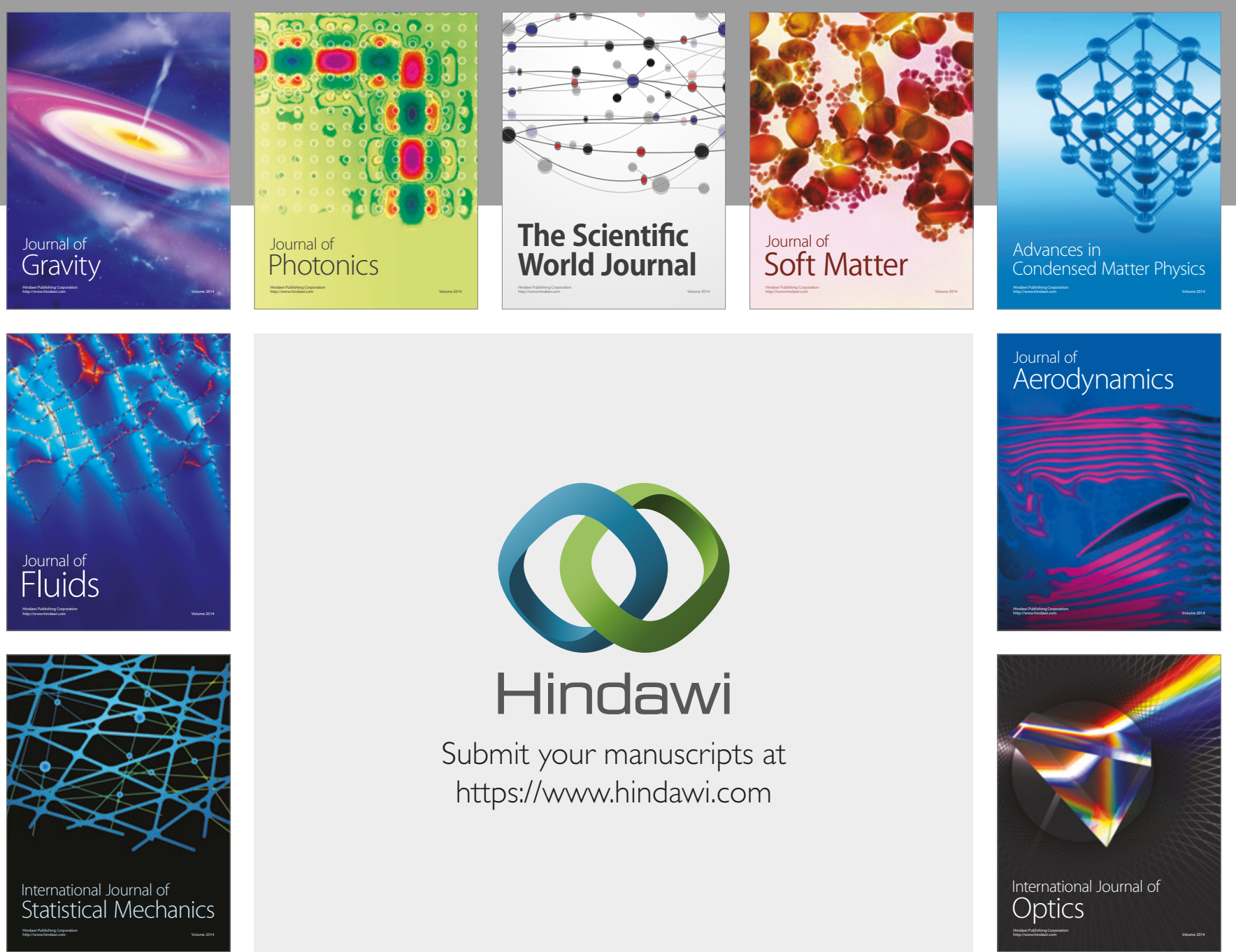

Submit your manuscripts at

https://www.hindawi.com
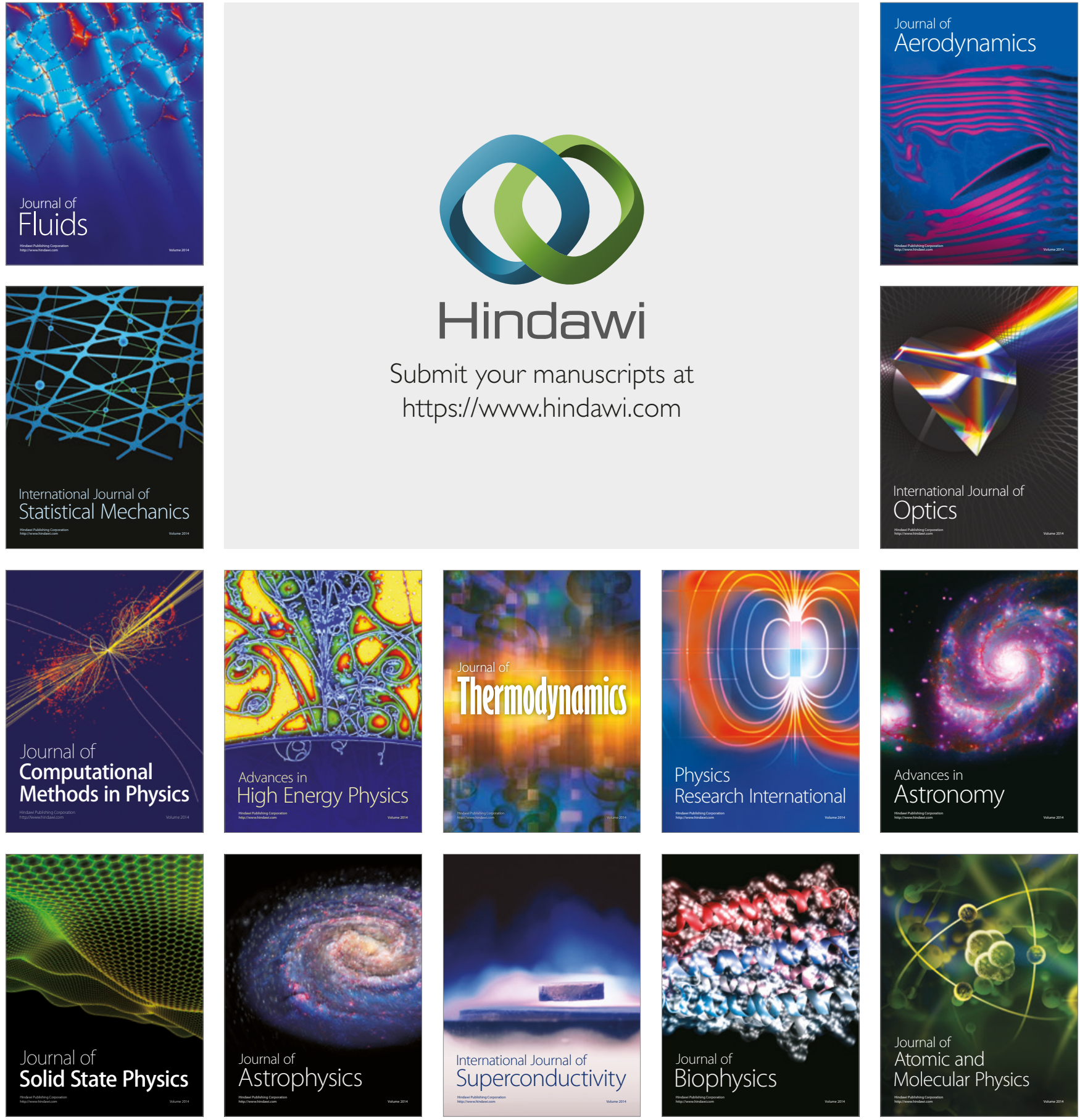